\title{
Nuclear data for advanced nuclear systems
}

\author{
V. Wagner', M. Suchopár, O. Svoboda, J. Vrzalová, P. Chudoba, P. Tichý, A. Kugler \\ Nuclear Physics Institute of the ASCR, v. v. $i$. \\ $\check{R} e z ̌$ 130, $25068 \check{R} e z ̌$, Czech Republic \\ E-mail: wagner@ujf.cas.cz, suchopar@ujf.cas.cz, svoboda@ujf.cas.cz, \\ vrzalova@ujf.cas.cz, chudoba@ujf.cas.cz, tichy@ujf.cas.cz, \\ kugler@ujf.cas.cz
}

\section{J. Adam, L. Závorka, A. Baldin, W. Furman, M. Kadykov, J. Khushvaktov, Yu. Kish, A. Solnyshkin, V. Tsoupko-Sitnikov, S. Tyutyunnikov}

Joint Institute for Nuclear Research

Joint Curie str. 6, 1419980 Dubna, Moscow region, Russia, Country

E-mail: iadamejinr.ru, zavorka@jinr.ru, Anton.Baldin@sunhe.jinr.ru,

furmanejinr.ru, kadykovejinr.ru, khushvaktovejinr.ru,kishyura@jinr.ru, soln@sunhe.jinr.ru, vtsoupko@jinr.ru, tsiesunhe.jinr.ru

\section{S. Kilim, M. Bielewicz, E. Strugalska-Gola, M. Szuta}

National Centre for Nuclear Research,

Otwock-Swierk 05-400, Poland

E-mail: s.kilimencbj.gov.pl, m.bielewicz@ncbj.gov.pl, elasgencbj.gov.pl, mszuta@ncbj.gov.pl

The development of advanced nuclear systems as generation IV reactors, accelerator driven systems and fusion reactors needs new reliable high quality nuclear data. The relativistic proton and light ion accelerator Nuclotron at JINR Dubna was used to study cross-sections of deuteron reactions on copper. The obtained excitation functions of different radionuclide productions by relativistic deuterons on copper were used for determination of beam integral during last experiment with E+T set-up using copper foil. The discrepancy which we had between beam integral values obtained by means of two aluminium foil monitors was resolved by this new analysis. The accurate cross-sections of neutron reactions in wide energy range and relativistic light ion reactions are the most important part of the experimental studies. We used two quasi mono-energetic neutron sources for studies of neutron reaction cross-sections on broad set of materials. The first facility is the neutron source based on the cyclotron at the Nuclear Physics Institute of ASCR, Řež. It provides neutron beams in the energy range from $14 \mathrm{MeV}$ up to 35 $\mathrm{MeV}$. The second neutron source is built around the cyclotron at TSL Uppsala. This facility provides neutron beam in the energy range from $14 \mathrm{MeV}$ up to $200 \mathrm{MeV}$. Both facilities are open for European users within the framework of project CHANDA.

XXII International Baldin Seminar on High Energy Physics Problems

15-20 September 2014

JINR, Dubna, Russia

${ }^{1}$ Speaker 


\section{Introduction}

The description of spallation reactions and induced neutron production needs precise information about differential cross-sections of relativistic proton and deuteron reactions. The reaction cross-sections of relativistic deuterons on copper are almost unknown. The copper and aluminium foils together are the important tools for beam integral determination by the activation method. We studied excitation functions of the different radionuclide productions on natural copper foils by means of activation analysis methods.

The activation detectors are also very useful method for determination of neutron production and transport inside different Accelerator Driven Transmutation (ADT) set-ups. They are very small and simple. It is possible to place such detectors in any position in the set-up. The knowledge about some excitation functions of mainly high threshold neutron reactions is very incomplete, even totally missing. We have started extensive project of neutron reaction cross-sections measurements of these reasons. We concentrated mainly on the yttrium which is the new useful activation detector material just now. Such studies are part of our common Accelerator Driven Transmutation (ADT) system research within Energy plus Transmutation (E\&T) of RAW collaboration.

\section{Relativistic deuteron reactions on copper}

The description of spallation reaction needs reliable Monte Carlo models and accurate data in cross-section libraries. Experimental data on excitation functions of relativistic deuterons are missing. A few values were measured for the relativistic deuteron reactions on the aluminium but only one value is known for the copper. This was reason for us to start studies of relativistic deuteron reactions on copper. First results of this research were presented during last Baldin seminar [1] in 2012.

\subsection{Differential cross-sections of different radionuclide production}

The systematic studies of these deuteron reactions on copper were completed during past years. The measurements were performed in the frame of the E\&T RAW collaboration during irradiations of QUINTA and GAMMA-3 set-ups with deuterons in JINR Dubna [2, 3]. The deuteron beams with energies ranging from $1 \mathrm{GeV}$ up to $8 \mathrm{GeV}$ were produced by JINR Nuclotron accelerator. Sixteen irradiations were performed during five sets of experiments carried out from 2011 to 2013. The activation method and gamma-ray spectrometry were used for the cross-section determination. Several high purity germanium detectors and more different geometries were used. Every radioactive sample was measured repeatedly to detect and identify short lived as well as long lived radioisotopes.

Very complex analysis of decay curves was necessary to distinguish separate radionuclides in the case of decay to the same daughter nucleus or decay sequences and when studying isomeric state population (for example ${ }^{44 \mathrm{~m}} \mathrm{Sc} \rightarrow{ }^{44} \mathrm{Sc} \rightarrow{ }^{44} \mathrm{Ti}$ in figure 1).

Overall 38 different radioisotopes were identified by their respective gamma lines (namely ${ }^{7} \mathrm{Be},{ }^{22} \mathrm{Na},{ }^{24} \mathrm{Na},{ }^{28} \mathrm{Mg},{ }^{28} \mathrm{Al},{ }^{38} \mathrm{~S},{ }^{38} \mathrm{Cl},{ }^{39} \mathrm{Cl},{ }^{42} \mathrm{~K},{ }^{43} \mathrm{~K},{ }^{47} \mathrm{Ca},{ }^{43} \mathrm{Sc},{ }^{44} \mathrm{Sc},{ }^{44 \mathrm{~m}} \mathrm{Sc},{ }^{46} \mathrm{Sc},{ }^{47} \mathrm{Sc},{ }^{48} \mathrm{Sc},{ }^{48} \mathrm{~V}$, ${ }^{48} \mathrm{Cr},{ }^{49} \mathrm{Cr},{ }^{51} \mathrm{Cr},{ }^{52} \mathrm{Mn},{ }^{54} \mathrm{Mn},{ }^{56} \mathrm{Mn},{ }^{52} \mathrm{Fe},{ }^{59} \mathrm{Fe},{ }^{55} \mathrm{Co},{ }^{56} \mathrm{Co},{ }^{57} \mathrm{Co},{ }^{58} \mathrm{Co},{ }^{60} \mathrm{Co},{ }^{56} \mathrm{Ni},{ }^{57} \mathrm{Ni},{ }^{65} \mathrm{Ni},{ }^{61} \mathrm{Cu}$, ${ }^{64} \mathrm{Cu},{ }^{62} \mathrm{Zn}$, and ${ }^{65} \mathrm{Zn}$ ) with half-life span from less than 1 hour to hundreds of days and their cumulative production cross-sections were measured using activation method. 


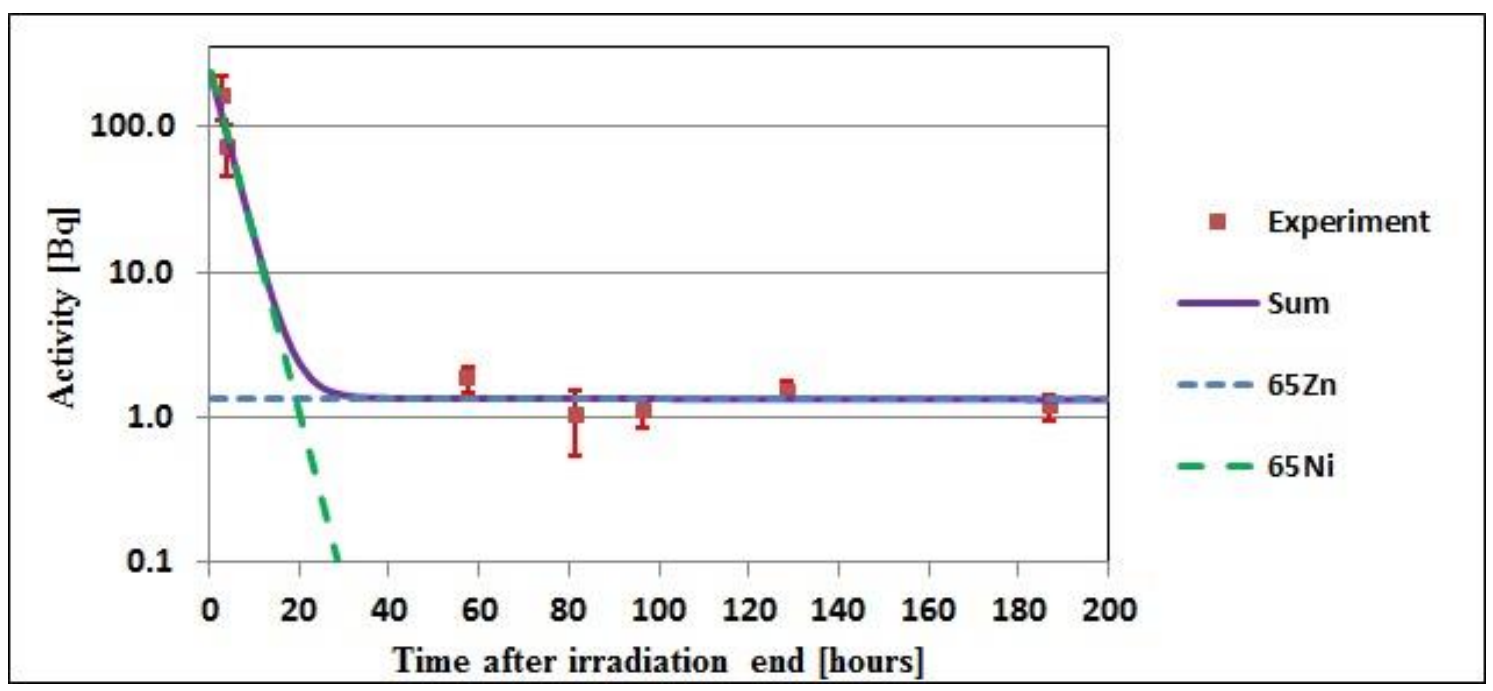

Fig. 1) Gamma decay curve of ${ }^{65} \mathrm{Cu}$ gamma lines (from $4 \mathrm{GeV}$ deuteron irradiation in December 2012),

More than one irradiation was done for selected energies. For instance, we obtained five independent values of cross-sections for deuteron energy $4 \mathrm{GeV}$ and three values for energy $2 \mathrm{GeV}$. Systematic uncertainties of the beam integral determination could be estimated by means of these data. Detailed analysis of different sources of systematic uncertainties was performed. It has to be noted that all measurements with $4 \mathrm{GeV}$ beam are inside $20 \%$ range and within expected uncertainties of individual irradiations (see figure 2). Similar situation and even better occurred for other deuteron energies which were measured more times.

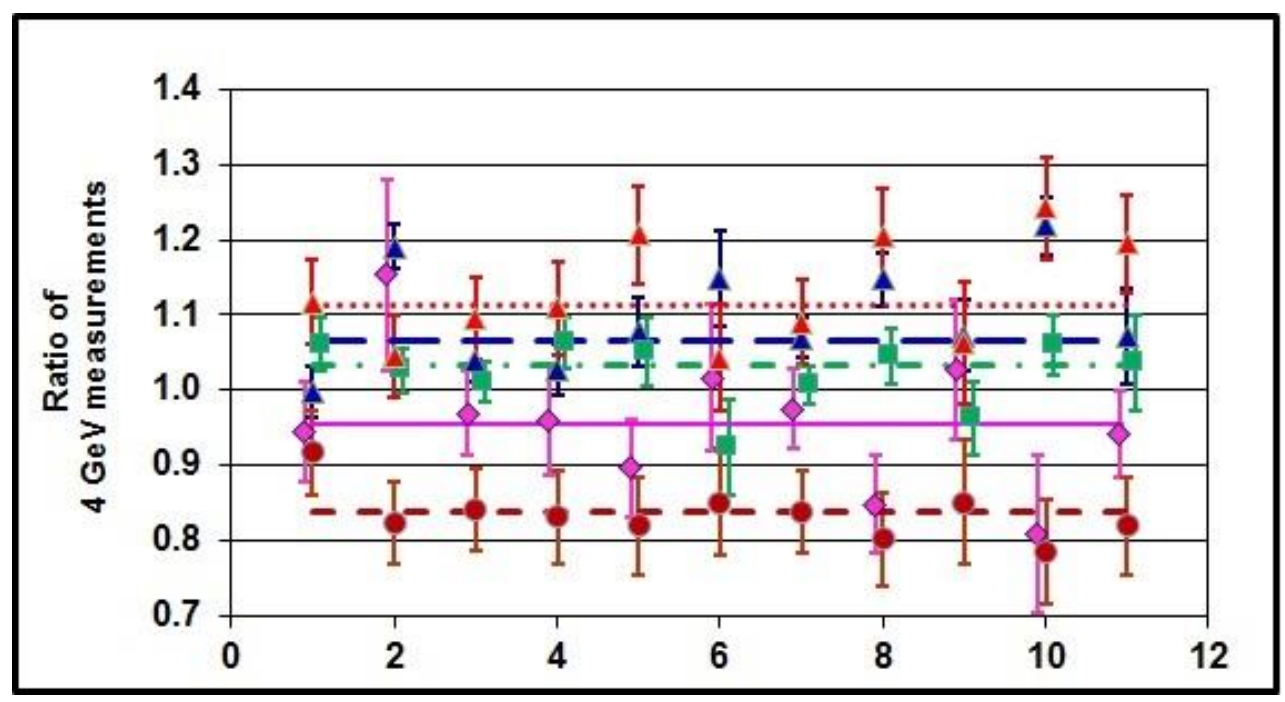

Fig. 2) Ratios of results obtained during five different experiments with $4 \mathrm{GeV}$ deuterons irradiations (March 2011 - violet rhomb, December 2011 blue triangle, March 2012 - green square, December 2012 - brown circle, March 2013 red triangle). Order of radionuclides is ${ }^{58}$ Co, ${ }^{56} \mathrm{Co},{ }^{52} \mathrm{Mn},{ }^{48} \mathrm{Sc},{ }^{44 m} \mathrm{Sc},{ }^{57} \mathrm{Ni},{ }^{48} \mathrm{~V},{ }^{47} \mathrm{Sc},{ }^{55} \mathrm{Co},{ }^{48} \mathrm{Cr}$ and ${ }^{43} \mathrm{~K}$. 
Values of different measured partial cross-sections extend over two orders of magnitude. They vary from maximal values of about tens of mbarn through values of around a few milibarns down to a fraction of milibarn. Most of our measured cross-sections are cumulative. We compared our new deuteron data with proton data from EXFOR library. The absolute values of the relativistic deuteron reaction partial cross-sections are higher mostly by about 30-40\% than those for proton reactions. Exception is the case of ${ }^{7} \mathrm{Be}$ production where cross-sections of deuteron reactions are about two times higher. The only one measured cross-section value of deuteron production of ${ }^{24} \mathrm{Na}$ on copper from the EXFOR database agrees nicely with our new data. The detailed description of these studies was published in [4] and the results are discussed in M. Suchopár et al. contribution in these proceedings.

\subsection{First practical usage of newly obtained excitation functions}

We made detailed systematic studies of E+T set-up consisting of lead target and uranium blanket irradiated by relativistic protons. We analysed neutron production and transmutation of actinides and fission products by means of gamma spectroscopy $[5,6]$. The same study was done also for deuteron beam. Two different aluminium beam monitors are used during every irradiation. Both are placed in different positions, measured by different gamma detectors and analysed independently. The corresponding results for both monitors were obtained for all previous irradiations.

However two independently obtained beam integral values were very different during 4 $\mathrm{GeV}$ deuteron experiment with E+T set-up in December 2009. First value was $(1.985 \pm 0.019) \cdot 10^{13}$ deuterons (only statistical uncertainties are given) [7] and second value was $(1.37 \pm 0.19) \cdot 10^{13}$ deuterons [8]. The situation was temporarily resolved by usage of average of these two values and sufficient uncertainty $(1.59 \pm 0.31) \cdot 10^{13}$ deuterons in the article [9].

Table 1: Cross-sections of deuterons with energy $4 \mathrm{GeV}$ on copper from our NIM B paper [2].

\begin{tabular}{|l|l|l|l|l|l|l|}
\hline $\begin{array}{l}\text { Pro- } \\
\text { duced } \\
\text { nuclide }\end{array}$ & $\begin{array}{l}\text { March } \\
2011 \\
{[\text { mbarn }]}\end{array}$ & $\begin{array}{l}\text { December } \\
2011 \\
{[\text { mbarn] }}\end{array}$ & $\begin{array}{l}\text { March } \\
2012 \\
\text { [mbarn] }\end{array}$ & $\begin{array}{l}\text { December } \\
2012 \\
\text { [mbarn] }\end{array}$ & $\begin{array}{l}\text { March } \\
2013 \\
\text { [mbarn] }\end{array}$ & $\begin{array}{l}\text { Average } \\
\text { value } \\
\text { [mbarn] }\end{array}$ \\
\hline${ }^{43} \mathrm{~K}$ & $2.07(8)$ & $2.12(6)$ & $2.11(13)$ & $2.06(11)$ & $2.26(12)$ & $2.11(4)$ \\
\hline${ }^{44 m} \mathrm{Sc}$ & $7.0(3)$ & $7.52(21)$ & $7.6(3)$ & $7.3(4)$ & $8.1(4)$ & $7.47(18)$ \\
\hline${ }^{47} \mathrm{Sc}$ & $3.38(16)$ & $4.09(12)$ & $3.85(13)$ & $3.63(18)$ & $4.12(21)$ & $3.84(26)$ \\
\hline${ }^{48} \mathrm{Sc}$ & $0.90(5)$ & $0.87(3)$ & $0.93(3)$ & $0.89(4)$ & $0.90(5)$ & $0.90(2)$ \\
\hline${ }^{48} \mathrm{Cr}$ & $0.355(26)$ & $0.48(3)$ & $0.430(16)$ & $0.392(17)$ & $0.467(26)$ & $0.42(4)$ \\
\hline${ }^{48} \mathrm{~V}$ & $11.9(9)$ & $11.7(3)$ & $11.4(3)$ & $11.7(6)$ & $11.4(6)$ & $11.56(19)$ \\
\hline${ }^{52} \mathrm{Mn}$ & $7.8(4)$ & $7.49(20)$ & $7.53(20)$ & $7.7(4)$ & $7.6(4)$ & $7.56(12)$ \\
\hline${ }^{55} \mathrm{Co}$ & $1.27(10)$ & $1.19(3)$ & $1.10(5)$ & $1.20(6)$ & $1.13(9)$ & $1.17(2)$ \\
\hline${ }^{56} \mathrm{Co}$ & $9.3(5)$ & $8.5(9)$ & $7.61(22)$ & $7.5(4)$ & $7.2(4)$ & $7.7(5)$ \\
\hline${ }^{58} \mathrm{Co}$ & $28.2(10)$ & $26.7(12)$ & $29.3(10)$ & $31.1(16)$ & $28.6(13)$ & $28.6(7)$ \\
\hline
\end{tabular}

To resolve satisfactorily this discrepancy, some independent beam monitor was needed. Our copper monitor was possible way to obtain new independent value of the beam integral in this case. The copper foil was placed near the target. Problem was that needed cross-sections of 
relativistic deuterons on copper were unknown up to now. The detailed study of such crosssections described in previous section was done by us during last four years and the obtained results were published in the NIM B article [4]. We used these published cross-sections for $4 \mathrm{GeV}$, see the table 1.

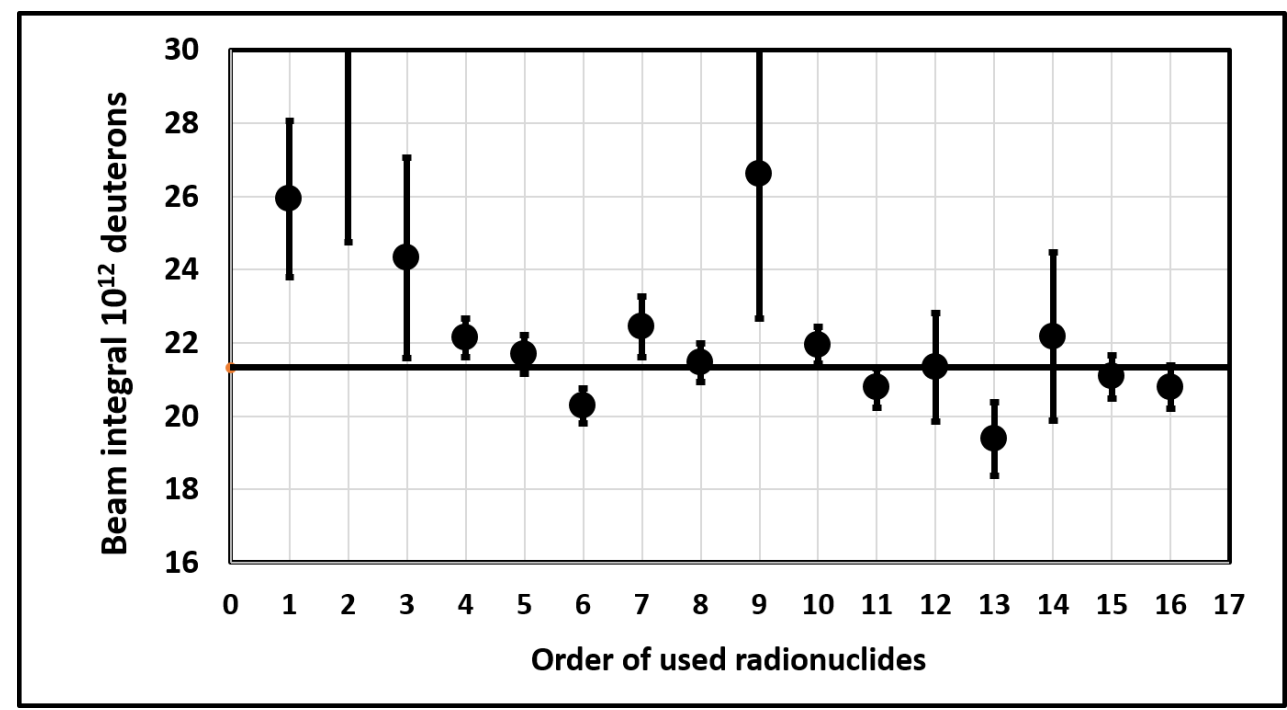

Fig. 3) The beam integral determined by different radionuclides produced in copper foil. Order of radionuclides is ${ }^{58} \mathrm{Co}(810 \mathrm{keV}),{ }^{56} \mathrm{Co}(847 \mathrm{keV}),{ }^{56} \mathrm{Co}(1238 \mathrm{keV}),{ }^{48} \mathrm{Cr}(112 \mathrm{keV}),{ }^{52} \mathrm{Mn}(1434$ $\mathrm{keV}),{ }^{52} \mathrm{Mn}(936 \mathrm{keV}),{ }^{52} \mathrm{Mn}(744 \mathrm{keV}),{ }^{48} \mathrm{Sc}(1038 \mathrm{keV}),{ }^{44 m} \mathrm{Sc}(271 \mathrm{keV}),{ }^{57} \mathrm{Ni}(1377 \mathrm{keV}),{ }^{48} \mathrm{~V}(983$ $\mathrm{keV}),{ }^{48} \mathrm{~V}(1312 \mathrm{keV}),{ }^{47} \mathrm{Sc}(159 \mathrm{keV}),{ }^{55} \mathrm{Co}(931 \mathrm{keV}),{ }^{48} \mathrm{Cr}(308 \mathrm{keV}),{ }^{43} \mathrm{~K}(373 \mathrm{keV})$ and ${ }^{43} \mathrm{~K}(617$ $\mathrm{keV})$.

The shape of beam was relatively sharp but very close to the boundary of our copper foil during this irradiation. The shape of the beam spot was determined by track detectors and also by cutting copper foil. It is possible that some part of deuterons was outside of our copper foil but such lost fraction of the beam was probably very small. We determined sixteen values of beam integral from production of different radionuclides, see figure 3 . Weighted average value is $(2.13 \pm 0.03) \cdot 10^{13}$ deuterons. The copper foil was in different place than the aluminium foil. The size of the copper foil was smaller $\left(8 \times 8 \mathrm{~cm}^{2}\right)$ than the size of the aluminium foil $\left(10 \times 10 \mathrm{~cm}^{2}\right)$. Distance between foils was a few meters and beam positions and shapes might be different. Also fraction of beam deuterons outside the foil might be different but it is mostly small (negligible according to beam spot determination). Main tendency should be to obtain smaller integral from smaller copper foil. Our value obtained from the copper foil is close to the first value obtained from the aluminium foil. It is slightly higher but within the systematic uncertainties estimated from comparison of values obtained from smaller copper foil and aluminium foil during other E+T and KVINTA experiments (about $10 \%$ ). The value $(1.99 \pm 0.20) \cdot 10^{13}$ deuterons from aluminium foil confirmed by the copper foil result will be used in the prepared articles about results from this irradiation (systematic uncertainties are included). 


\section{Neutron reactions important for ADT system studies}

The activation detectors are used to study neutron flux produced inside ADT set-ups. The cross-sections of threshold neutron reactions with materials used as activation detectors were missing in some energy regions up to now. We performed extensive studies of excitation functions of neutron reactions by means of two quasi mono-energetic neutron sources based on the proton beam provided by cyclotron and lithium target. The mentioned neutron sources are placed at the NPI Řež and TSL Uppsala [10, 11]. The first review of our results was published in NIM A article [12]. We concentrated on study of threshold neutron reactions with the yttrium later. This material is very promising as activation detectors but well measured excitation functions are still missing. Our measurements of these reactions are presented in P. Chudoba et al. contribution in these proceedings.

\section{Conclusions and outlooks}

The very important part of ADT system studies is measurement of excitation functions of important neutron and relativistic particle reactions. We measured the cross-sections of relativistic deuterons with natural copper. The obtained results made possible to use the copper foil as relativistic deuteron beam monitor. We confirmed one of beam integral values obtained by the two different aluminium monitors during last experiment with the E+T set-up consisting of lead target and uranium blanket in 2009.

The comparison of the obtained deuteron excitation functions with known proton ones would be possible. The improvement of spallation reaction models and codes would be also possible.

The extensive set of neutron reaction cross-section data was obtained. We used two quasi mono-energetic neutron sources and broad energy range of neutrons from $14 \mathrm{MeV}$ up to $100 \mathrm{MeV}$. Such successful studies were possible because of projects of transnational access of European users to European neutron sources. We used support of projects EFNUDAT and ERINDA in previous years. The new studies on neutron source at TSL Uppsala were performed during beginning of January 2015. These experiments were supported by new project CHANDA. We have already started analysis of newly obtained data. The project CHANDA is open for European users and the $\check{R}$ ž and the Uppsala sources are open for experiments.

\section{Acknowledgements}

This work was supported by the ̌̌ež-Dubna funds, CANAM project and ERINDA program. The authors are grateful to the staff of the Dubna Nuclotron accelerator for providing a good deuteron beam and to the staff of TSL Uppsala and NPI Řež for neutron beams. We would also like to thank Pavel Bém, Eva Šimečková and Mitja Majerle for the possibility of participation in their irradiations.

\section{References}

[1] V. Wagner, J. Vrzalová, M. Suchopár et al, Studies of deuteron and neutron cross-sections important for ADS research, Proceedings of science (Baldin ISHEPP XXI) 090

[2] W. Furman et al, Recent results of the study of ADS with $500 \mathrm{~kg}$ natural uranium target assembly QUINTA irradiated by deuterons with energies from 1 to $8 \mathrm{GeV}$ at JINR NUCLOTRON, Proceedings of Science (Baldin ISHEPP XXI 086)

[http://pos.sissa.it/archive/conferences/173/086/Baldin\%20ISHEPP\%20XXI_086.pdf] 
[3] J. Adam, C. Bhatia, V. Kumar et al, A study of reaction rates of $(n, f),(n, g)$ and $(n, 2 n)$ reactions in $U$ nat and Th-232 by the neutron fluence produced in the graphite set-up (GAMMA-3) irradiated by 2.33 GeV deuteron beam, European Physical Journal A 47 (2011) 85

[4] M. Suchopár, V. Wagner, O. Svoboda et al, Cross-section studies of relativistic deuteron reactions on copper, Nuclear Instruments and Methods in Physics Research B 344 (2015) 63 - 69.

[5] M. I. Krivopustov et al, First experiments with a large uranium blanket within the installation "Energy plus Transmutation" exposed to $1.5 \mathrm{GeV}$ protons, Kerntechnik 68 (2003) 48-54

[6] A. Krása, V. Wagner, M. Majerle et al, Neutron production in a Pb/U-setup irradiated with 0,7-2.5 GeV protons and deuterons, Nuclear Instruments Methods in Physics Research A 615 (2010) 70 77.

[7] O. Svoboda, V. Wagner, D. Wagner, The Intensity and Shape of the $4 \mathrm{GeV}$ Deuteron Beam from JINR Nuclotron (November 2009) - Results from the Rež Group, internal publication for E+T collaboration, 2010

[8] W. Westmeier W. et al.: Experiment S: $4.00 \mathrm{GeV}$ deuterons on E+T, internal publication for E+T collaboration, 2011

[9] J.J. Borger et al: Spatial distribution of thorium fission rate in a fast spallation and fission neutron field: An experimental and Monte Carlo study, Nucl. Instr. And Meth. In Phys. Research A 664 (2012) 103-110

[10] Bém et al. - The NPI cyclotron-based fast neutron facility, Proceedings of the International Conference on Nuclear Data for Science and Technology - ND2007, Nice, France, (2007) 555-558.

[11] A. V. Prokofiev et al. - The TSL Neutron Beam Facility, Rad. Prot. Dos. 126 (2007) 18-22

[12] J.Vrzalová, O. Svoboda, A. Krása et al, Studies of (n,xn) cross-sections in Al, Au, Bi, Cu, Fe, I, In, $\mathrm{Mg}, \mathrm{Ni}, \mathrm{Ta}, \mathrm{Y}, \mathrm{Zn}$ by the activation method, Nuclear Instruments and Methods in Physics Research A 726 (2013) 84 - 90 